\title{
EVALUACIÓN Y SELECCIÓN DE LÍNEAS DE ARVEJA CON GEN AFILA BAJO DOS DENSIDADES DE POBLACIÓN
}

\section{EVALUATION AND SELECTION OF PEA LINES WITH AFILA GENE IN TWO PLANTING DENSITIES}

\author{
Marcela Elizabeth Riascos Delgado ${ }^{1}$, Oscar Eduardo Checa Coral ${ }^{2}$
}

${ }^{1}$ I.A., M.Sc. C.I. Obonuco, Agrosavia, Kilómetro 5, Vía Pasto-Obonuco, San Juan de Pasto-Nariño, Colombia, e-mail: meriascos@agrosavia.co, (iDhttp://orcid.org/0000-0003-0573-2748; ${ }^{2}$ I.A. Ph.D., Grupo de Investigación en Cultivos Andinos GRICAND, Facultad de Ciencias Agrícolas. Universidad de Nariño -sede Vipri-, Cra. 36 No. 5-121, bloque 5, oficina 408, San Juan de Pasto-Nariño, Colombia, e-mail: ocheca@udenar.edu.co, (iDhttp://orcid.org/0000-0002-6929-7717

\author{
Rev. U.D.C.A Act. \& Div. Cient.21(1):367-376, Julio-Diciembre 2018 \\ https://doi.org/10.31910/rudca.v21.n2.2018.984
}

\begin{abstract}
Artículo de acceso abierto publicado por Revista U.D.C.A Actualidad \& Divulgación Científica bajo una licencia Creative Commons CC BY-NC 4.0
\end{abstract}

\section{RESUMEN}

Nariño es el principal productor de arveja en Colombia, posición lograda por el uso de sistemas de tutorado y variedades mejoradas; sin embargo, estos genotipos presentan crecimiento agresivo y son de difícil manejo agronómico, lo cual, encarece los costos de producción. Como alternativa, el Grupo de Cultivos Andinos (GRINCAND), desarrolló un proceso de introducción del gen recesivo afila (af), en variedades comerciales (Andina, San Isidro y Sindamanoy), logrando reemplazar las hojas laterales por zarcillos, mejorando el agarre de las plantas. Las líneas de arveja afila resultantes fueron evaluadas en tres fases experimentales. En la primera fase, de 208 familias F3, se seleccionaron y generaron 105 líneas F4; luego, las 105 líneas se evaluaron agronómicamente y mediante la aplicación de un índice de selección, se escogieron por orden de mérito, basado en componentes de rendimiento y de reacción a enfermedades, 21 líneas promisorias. Estas líneas, se evaluaron bajo dos densidades de población (D1:83.333 y D2:166.666 plantas $\cdot \mathrm{ha}^{-1}$ ), utilizando un diseño experimental de parcelas divididas. La parcela principal correspondió a las densidades de población y, la subparcela, los genotipos de arveja. En D2, la línea SXDRC118, tuvo mayor rendimiento que los testigos, con una ganancia entre 75 y $211 \%$; además, esta línea superó al 66\% de las líneas evaluadas. SX3568RC17 mostró un promedio mayor sobre San Isidro y Sindamanoy, en 86 y 188\% y SX3575RC18, SX3575RC1X1 y SX3575RC19 sobre Sindamanoy, en 138 a 157\%. Esto indica que la siembra de algunas líneas afila, a mayor densidad, pueden superar el rendimiento de las variedades comerciales.
Palabras clave: zarcillos, retrocruzamiento, genotipos, selección, rendimiento.

\section{ABSTRACT}

Nariño is the principal pea producer in Colombia, given its position in the use of improved varieties and trellis system. However, these genotypes have difficult agronomic managements, because their aggressive growth, which increases production costs. As alternative, the group of investigation Cultivos Andinos (Gricand), developed a process of introduction afila (af) gene in the pea varieties Andina, San Isidro and Sindamanoy, replacing lateral leaves by tendrils, to get a better grip of plants. The lines afila resulted were evaluation in three experimental phases. In the first phase, 208 family F3 were selection and generated 105 lines F4. In following cycle, the lines selected were evaluated for yield components and applying a selection index, 21 lines promising for merit order were selected. Subsequently, these lines were evaluated in two planting densities (D1: 83.333 and D2: 166,666 plantas $\cdot \mathrm{ha}^{-1}$ ), used a splits plot design. The main plot with the densities and the subplot the pea genotypes. In the planting density D2, the line SXDRC118 had higher yield than the controls, with a gain between 75 and $211 \%$; furthermore, it surpassed with $66 \%$ the other lines. SX3568RC17 showed a higher average yield than San Isidro and Sindamanoy, respectively, 86 and $188 \%$ and SX3575RC18, SX3575RC1X1 and SX3575RC19 regarding Sindamanoy with 138 to $157 \%$. This indicates that some lines with af gene have potential for higher yield than commercial varieties with normal leaves when planted at higher density.

Keywords: tendrils, backcross, genotypes, selection, yield. 


\section{INTRODUCCIÓN}

El cultivo de arveja en Nariño representa una de las principales fuentes de empleo en el campo (Fenalce, 2010). Checa et al. (2017) indican que las variedades de arveja más utilizadas en Nariño son: Obonuco Andina, Obonuco San Isidro y Sureña, que requieren de sistemas de tutorado, para lograr mejor aprovechamiento del potencial genético. El uso de estas variedades y sistemas de cultivo han permitido que las áreas dedicadas a la producción de arveja en Nariño pasen de 3.500 hectáreas, antes de 1995, a 13.363, en el 2016, posicionado el producto en el mercado del interior del país.

En arveja voluble las plantas, a pesar de estar tutoradas verticalmente, se tienden sobre el suelo, debido a su crecimiento agresivo, especialmente, en la época de llenado de grano, siendo necesario retutorar horizontalmente, para facilitar las labores de campo y mantener un cultivo sano; sin embargo, la necesidad de combinar el tutorado horizontal y vertical implica mayores gastos en mano de obra e insumos, que representa el $52 \%$ de los costos totales de producción (Checa \& Rodríguez, 2015; Checa, 2014).

Con el fin de obtener líneas con mejor arquitectura de planta, aumentado el número de zarcillos y lograr un mejor agarre en los sistemas de tutorado, el grupo de Investigación de Cultivos Andinos (Gricand) de la Universidad de Nariño, realizó cruzamientos y retrocruzamientos entre las variedades Andina, San Isidro y Sindamanoy, con los genotipos tipo afila de crecimiento determinado, ILS3568, ILS3575 y la variedad Dove. El objetivo de hacer retrocruzamiento entre estos genotipos, está encaminado en ganar el gen afila conservando gran parte de las características de las variedades más aceptadas en el departamento de Nariño.

El gen afila (af) es un gen recesivo que reemplaza los foliolos laterales por numerosos zarcillos, conservando únicamente las estípulas, que abrazan el tallo (Hofer et al. 2009). La abundancia en la cantidad de zarcillos permite que las plantas se mantengan erectas, se apoyen entre sí y mejoren su capacidad de aprovechamiento de hilos, que les sirve como tutor, además de configurar una estructura abierta, que da buena aireación a la planta (Giaconi \& Escafe, 1988).

En países, como Holanda, España y Francia, desde 1986, se han registrado las variedades comerciales con gen afila Ascona, Ballet, Cea, Elsa, Amadeus, Charleston, Alex, Choque y Astuce, que presentan rendimientos similares a las variedades de follaje normal (Mera et al. 1996). En Chile, la variedad de arveja con afila INIA Brisca presentó rendimiento similar a la variedad comercial Solara (Mera et al. 1996). Pesic et al. (2013) y Dordević et al. (2004) reportaron el uso de la variedad Filigreen, de origen inglés, como padre donante del gen afila. En Colombia, no existen cultivares registrados con gen afila.

La densidad de población es un factor determinante en el rendimiento del cultivo arveja voluble convencional. A medida que aumenta la densidad, las plantas son individualmente menos productivas (Mera et al. 1996). En Colombia, se establecen las densidades de población para arveja, de acuerdo con el hábito de crecimiento de la variedad; para arbustivas, se recomienda densidades de 60 a $80 \mathrm{~kg} \cdot \mathrm{ha}^{-1}$ de semilla (Checa, 1994) y, en genotipos de arveja volubles convencionales, 30 a $35 \mathrm{~kg} \cdot \mathrm{ha}^{-1}$. Para los genotipos afila no se tiene datos relacionados con la densidad poblacional, la cual, debe ser ajustada, ya que presenta una arquitectura nueva y una menor área foliar. Obtener y aprovechar el potencial genético de líneas con gen af y de alto rendimiento, se puede convertir en una alternativa productiva para lograr mayor eficiencia y rentabilidad. Por lo anterior, en la presente investigación, se evaluaron y seleccionaron, bajo dos densidades de población, líneas promisorias de arveja con gen af, obtenidas por retrocruzamiento.

\section{MATERIALES Y MÉTODOS}

La investigación, se realizó en las instalaciones del Centro Internacional de Producción Limpia-Lope del Servicio Nacional de Aprendizaje -SENA, ubicado en el municipio de Pasto (Nariño, Colombia), a una altura de $2.633 \mathrm{~m}$ s.n.m.,

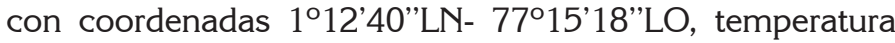
promedia de $13^{\circ} \mathrm{C}$, precipitación promedia de $700 \mathrm{~mm} / \mathrm{año}$ y humedad relativa entre 60 y $80 \%$

El material genético correspondió a 208 familias F3, generadas a partir de retrocruzamientos entre las variedades mejoradas de arveja Andina, San Isidro y Sindamanoy (padres recurrentes) y los genotipos con gen afila Dove, ILS3575 e ILS3568 (padres donantes).

La investigación, se desarrolló en tres fases: en la primera fase, se sometieron a una selección visual las 208 familias F3, utilizando, como criterios de selección, plantas de porte erecto, entrenudos cortos, altura mediana (entre 1 y $2.50 \mathrm{~m}$ ), estípulas grandes, alto número de vainas por planta y un número de granos por vaina no menor a 6 .

Dentro de las familias de mejor comportamiento, se seleccionaron 105 plantas individuales, cuya semilla constituye las líneas F4, que se utilizaron en la siguiente fase de evaluación. Las 105 líneas F4 con gen afila seleccionadas, se llevaron a una prueba de evaluación preliminar, que incluyó las variables días a cosecha en vaina verde (DCVV), altura de planta (ALT), número de estipulas (NEST), largo de entrenudo (LENT), área de estipula (AEST), largo de vaina (LV), peso de vaina verde $(\mathrm{PVV})$, relación Grano/vaina $(\mathrm{G} / \mathrm{V})$, número 
de granos por vaina (NGV), peso de cien granos verdes (PCGV) y evaluación por enfermedades Ascochyta pisi (ASCH) y Eryshipe pisi, (OIDIO), para lo cual, se adaptaron las escalas de severidad de Orbes \& Becerra (1982) y Falloon et al. (1989).

El orden de mérito de las líneas evaluadas, se estableció utilizando un índice de selección estandarizado, dando diferentes pesos a las variables en estudio, con base en su importancia, desde el punto de vista de la capacidad productiva, la calidad y la sanidad del cultivo. Para evitar el efecto de las unidades de medida de las variables sobre el índice, los datos fueron previamente estandarizados. Para la variable ALT, se clasificó en una escala de 1 a 5 , de la siguiente forma: $1=<1 \mathrm{~m} ; 2=$ $>1<1,49 \mathrm{~m} ; 3=>2,5 \mathrm{~m} ; 4=>2,1<2,5 \mathrm{~m}$ y $5=>1,50<$ $2 \mathrm{~m}$, siendo este último rango el más deseable.

Con una presión de selección del 20\%, el índice de selección se calculó de la siguiente manera:

Índice de selección (IS) $=\left(\mathrm{X}_{\text {STD DCVV }}\right) \times 6+\left(\mathrm{XSTD}_{\mathrm{STD}} \mathrm{ALT}\right) \times 7$ $+\left(\mathrm{X}_{\text {stD }}\right.$ NEST $) \times 7+\left(\mathrm{X}_{\text {stD }}\right.$ LENT) $\times 5+\left(\mathrm{X}_{\text {stD }}\right.$ AEST) $\times 7+$ (XStD LV) $x 7+\left(X_{\text {StD }}\right.$ PVV) $\times 10+($ XStD G/V) $x 10+$ XstD NGV) $9+\left(\mathrm{X}_{\text {STD }}\right.$ PCGV) $\times 9+\left(\mathrm{X}_{\text {StD }}\right.$ ASCH $) \times 5+\left(\mathrm{XSTD}_{\text {STD }}\right.$ OIDIO $) \times 5$, en donde, $\mathrm{X}_{\mathrm{STD}}$ corresponde al promedio estandarizado de cada variable.

Una vez aplicado el índice de selección, se escogieron 21 líneas F5, para la tercera fase del estudio, evaluándolas por rendimiento y sus componentes, mediante un diseño de bloque al azar, con arreglo en parcelas, divididas con tres repeticiones, en donde la parcela principal correspondió a dos densidades de población (166.666 y 83.333plantas $\cdot$ ha $^{-1}$ ) y, la subparcela, a las 21 líneas con gen af y tres testigos comerciales (Andina, San Isidro y Sindamanoy). Para esta generación (F5), se evaluaron las variables ALT, DCVV, NEST, LV, NGV, PVV, G/V y PCGV, antes descritas en la evaluación de la generación $\mathrm{F} 4$, adicionando las variables días a floración (DF), número de vainas por planta (NVP) y rendimiento en vaina verde (REND).

Los resultados, se sometieron a análisis de varianza y prueba de comparación de promedios de Duncan, utilizando el software SAS 9.4.

\section{RESULTADOS Y DISCUSIÓN}

Selección de líneas en generación F4: Las 21 líneas fueron seleccionadas por aplicación de un índice de selección estandarizado, en 105 líneas de la F4. Dentro de ellas, la línea SX3575RC112, con 107 días de cosecha en vaina verde (DCVV), se mostró como el material más precoz (Tabla 1). El $71 \%$ de las líneas seleccionadas necesitaron 112 días para cosecha en vaina verde, mientras que tres fueron más tardías, con 118 días. Para altura de planta (ALT), los valores de las 21 líneas seleccionadas mostraron alturas entre 1,5 y 2,5m (en la escala valores de 4 y 5). En cuanto al largo de entrenudo (LENTRE), la variación observada en las líneas seleccionadas estuvo en un rango comprendido entre 10,6 y $16,1 \mathrm{~cm}$; la altura de la planta está determinada por el número total de nudos, establecidos por los genes miu mie, min; además, otro factor que condiciona la altura es la distancia entre los nudos, la que es de naturaleza compleja y dependiente del ambiente (Khvostova, 1983). Las líneas con mayor altura, se pueden ver favorecidas en rendimiento, porque en plantas más altas es mayor el número de nudos reproductivos (Khvostova, 1983; Burstin et al. 2007); no obstante, las plantas con alturas superiores a $2 \mathrm{~m}$ incrementan los costos de actividades, como el tutorado y la cosecha (Pacheco et al. 2011), razón, por la cual, en la escala de altura no se le dio los mayores valores a las plantas que superan los $2 \mathrm{~m}$. Por otra parte, las 21 líneas seleccionadas presentaron entre 20 y 28 estípulas por planta (NEST), con un área foliar (AEST) que osciló entre 46,8 y $151,7 \mathrm{~cm}^{2}$. Las líneas tipo afila seleccionadas cuentan con un número representativo de estípulas, característica de interés, porque la modificación de hojas por zarcillos puede reducir el área foliar y corresponde a las estípulas cumplir en mayor grado la función fotosintética, en ausencia de los foliolos laterales (Cote et al. 1992).

En las líneas seleccionadas, el peso de vaina (PVV) fluctúo entre 5,5 y 10g. De acuerdo con Hofer et al. (1997) y Cote et al. (1992), la reducción del área foliar en este tipo de plantas no afecta la floración ni la capacidad de producir vainas, por tanto, los guisantes tipo afila pueden presentar igual o mayor productividad que los cultivares convencionales, por la captación de luz que se presenta en órganos, como las estipulas, zarcillos jóvenes y los tallos.

Para Ascochyta pisi (ASCH), las líneas seleccionadas reaccionaron al inoculo natural, en un rango de resistente a moderadamente resistente. De igual forma, para Erysiphe pisi (OIDIO) mostraron una reacción de campo, entre moderadamente resistentes y altamente resistentes.

De las 21 líneas seleccionadas, 19 provienen de la generación F4RC1 de los cruzamientos Sindamanoy x ILS3575, Sindamanoy x ILS3568, San Isidro x Dove y Sindamanoy x Dove, con una recuperación del $75 \%$ del padre recurrente, correspondiente a las variedades Andina, Sindamanoy y San Isidro. Tan solo dos líneas fueron seleccionadas en la generación F4RC2, pertenecientes al cruzamiento Andina x Dove, donde existe una recuperación del padre recurrente (Andina), del 87,5\% (Tabla 1). Las variedades mejoradas que se utilizaron en el retrocruzamiento, como padres recurrentes Andina, Sindamanoy y San Isidro, presentan características deseables, especialmente, relacionadas con adaptación, tamaño de grano y rendimiento; sin embargo, en evaluaciones anteriores, realizadas por Checa \& Rodríguez (2015), obser- 
Tabla 1. Promedios de 21 líneas de arveja con gen afila seleccionadas por orden de mérito después de aplicar el índice de selección, a partir de 105 líneas F4.

\begin{tabular}{|l|r|r|r|r|r|r|r|r|r|r|r|r|r|}
\hline GENOTIPO & $\begin{array}{r}\text { DCV } \\
\text { (Día) }\end{array}$ & ALT & NEST & $\begin{array}{c}\text { LENTRE } \\
\mathbf{( c m})\end{array}$ & $\begin{array}{c}\text { AEST } \\
\left(\mathbf{c m}^{2}\right)\end{array}$ & $\begin{array}{c}\text { LV } \\
(\mathbf{c m})\end{array}$ & $\begin{array}{c}\text { PVV } \\
\mathbf{( g )}\end{array}$ & $\mathbf{G} / \mathbf{V}$ & NGV & $\begin{array}{c}\text { PCGV } \\
\mathbf{( g )}\end{array}$ & ASCH & OIDIO & IS \\
\hline SX3568RC17 & 112 & 4 & 20 & 11,5 & 96,4 & 8,9 & 10 & 0,6 & 8 & 76,1 & 2 & 2 & 68,93 \\
\hline SX3575RC19 & 112 & 4 & 29 & 11,3 & 50,2 & 9,5 & 8,6 & 0,6 & 7 & 43,4 & 1 & 3 & 64,03 \\
\hline SX3568RC19 & 112 & 4 & 21 & 12,2 & 77,8 & 9,7 & 8,5 & 0,7 & 7 & 77,6 & 2 & 2 & 61,25 \\
\hline SX3568RC11 & 112 & 4 & 22 & 12,4 & 58 & 7,5 & 7 & 0,9 & 7 & 92,4 & 3 & 2 & 57,01 \\
\hline SIXDRC1100 & 115 & 5 & 26 & 14 & 151,7 & 9,2 & 7,7 & 0,7 & 7 & 38,8 & 2 & 2 & 54,66 \\
\hline SX3568RC166 & 112 & 4 & 26 & 11,3 & 99,3 & 8,9 & 7,9 & 0,6 & 7 & 75,5 & 2 & 2 & 53,43 \\
\hline SIXDRC166 & 112 & 5 & 23 & 12,9 & 76,2 & 8,1 & 7,9 & 0,7 & 7 & 71,3 & 2 & 2 & 51,86 \\
\hline SX3575RC120 & 112 & 4 & 24 & 12,2 & 102,8 & 8,9 & 7,3 & 0,6 & 7 & 64,2 & 2 & 2 & 46,09 \\
\hline SX3575RC112 & 107 & 5 & 21 & 12,1 & 97,7 & 9,3 & 7,5 & 0,7 & 7 & 38,8 & 2 & 3 & 42,72 \\
\hline SIXDRC154 & 112 & 5 & 22 & 13,2 & 79,1 & 8,2 & 7 & 0,7 & 7 & 34,3 & 2 & 2 & 38,34 \\
\hline SIXDRC151 & 112 & 5 & 18 & 15,6 & 97,8 & 8,9 & 7,8 & 0,7 & 7 & 78,8 & 3 & 2 & 34,50 \\
\hline AXDRC215 & 112 & 5 & 24 & 11,8 & 99,9 & 8,3 & 6,3 & 0,6 & 6 & 45,9 & 1 & 1 & 29,13 \\
\hline SXDRC118 & 118 & 4 & 23 & 12,5 & 81,4 & 9,4 & 7,8 & 0,7 & 6 & 35,1 & 2 & 1 & 29,06 \\
\hline SX3575RC113 & 112 & 5 & 27 & 11,1 & 69,1 & 7,5 & 5,6 & 0,7 & 6 & 27,4 & 1 & 2 & 27,83 \\
\hline SX3575RC1X1 & 118 & 5 & 20 & 13,2 & 91,8 & 8,9 & 6,6 & 0,7 & 7 & 61,8 & 3 & 1 & 27,69 \\
\hline SX3568RC117 & 112 & 4 & 26 & 10,6 & 46,8 & 7,1 & 5,5 & 0,7 & 6 & 67,1 & 1 & 1 & 27,58 \\
\hline AXDRC24 & 112 & 5 & 23 & 11,7 & 94 & 7,6 & 6,1 & 0,6 & 7 & 51,9 & 1 & 3 & 25,43 \\
\hline SX3568RC128 & 112 & 4 & 26 & 11,9 & 79,5 & 9,4 & 6,9 & 0,7 & 6 & 42,7 & 2 & 2 & 24,45 \\
\hline SX3575RC18 & 112 & 4 & 25 & 13 & 88,3 & 8,5 & 6,7 & 0,7 & 7 & 73 & 3 & 3 & 21,46 \\
\hline SXDRC156 & 118 & 4 & 24 & 16,1 & 75,7 & 8,6 & 7 & 0,7 & 7 & 68,3 & 2 & 1 & 21,26 \\
\hline SIXDRC183 & 115 & 4 & 22 & 11 & 85,1 & 9,4 & 6,7 & 0,7 & 7 & 31 & 2 & 2 & 21,22 \\
\hline
\end{tabular}

DCVV: Días a cosecha en vaina verde; ALT: Altura de planta; NEST: Número de estipulas por planta; LENTRE: Largo de entrenudo; AEST: Área de estipula; LV: Largo de vaina; PVV: Peso de vaina verde; G/V: Relación grano/vaina; PCGV: Peso de cien granos verdes; ASCH: Evaluación de Ascochyta pisi; OIDIO: Evaluación de Eryshiphe pisi; IS: Índice de selección.

varon que algunas de las variables, como número de granos por vaina, puede ser mejorado en estas variedades. De igual manera, la altura de planta y relación grano/vaina no hacen parte de sus mejores atributos, por tanto, para estos rasgos, acercarse más al fenotipo de las variedades mejoradas, antes mencionadas, no resultaría deseable. En el índice de selección, se dio alto peso específico a estas variables, lo cual, afectó negativamente la selección de los genotipos, que provienen del retrocruzamiento dos (RC2).

Evaluación por rendimiento y sus componentes de 21 líneas con gen afila seleccionadas: En densidades de población, se presentaron diferencias significativas para NVP y altamente significativas para DF y REND. Para los genotipos evaluados, se presentó diferencias altamente significativas en LV, PVV, G/N, PCGV y REND. La interacción densidad por genotipo fue altamente significativa para NVP y significativa REND (Tabla 2).
La comparación de densidades de población para la variable DF presentó una diferencia de dos días, entre la densidad D1:83.333plantas' ha $^{-1}$, que floreció a los 56 días y la densidad D2:166.666plantas ha $^{-1}$, que lo hizo a los 54 días. El resultado sugiere que esta variable es ligeramente sensible a la densidad de población y a las condiciones ambientales, confirmando lo reportado por González \& Ligarreto (2006); los procesos fenológicos de la floración en condiciones de alta densidad, se pueden ver afectados por cambios en las condiciones ambientales. Estos resultados tienen la misma tendencia de los encontrados por Casanova et al. (2012), quienes reportaron que los genotipos sembrados a mayor densidad (666.666plantas ha $^{-1}$ ) mostraron una floración más precoz respecto a los de baja densidad (250.000plantas $\cdot$ ha $^{-1}$ ); no obstante, Azpilicueta et al. (2012) encontraron que, en densidades altas de 75, 105 y 125 plantas $\mathrm{m}^{2}$, no hubo diferencias para días a floración. Lo anterior, indica que, al pasar 
Tabla 2. Cuadrados medios para las variables: días a floración (DF), días a cosecha en vaina verde (DCVV), altura (ALT), número de estipulas (NESTT), largo de vaina (LV), número de grano por vaina (NGV), número de vainas por planta (NVP), peso de vaina verde $(\mathrm{PVV})$, relación grano/vaina $(\mathrm{G} / \mathrm{V})$, peso de cien granos verdes (PCGV) y rendimiento (REND).

\begin{tabular}{|c|c|c|c|c|c|c|c|}
\hline F de V & GL & DF & DCVV & ALT & NESTT & LV & NGV \\
\hline Repetición & 2 & 25,86 & 801,69 & 0,08 & 652,71 & 1,94 & 0,18 \\
\hline Densidad(D) & 1 & $128,44 * *$ & $31,17 \mathrm{~ns}$ & $0,006 \mathrm{~ns}$ & $126,56 \mathrm{~ns}$ & $3,21 \mathrm{~ns}$ & $0,11 \mathrm{~ns}$ \\
\hline Error a & 2 & 2,52 & 6,36 & 0,007 & 74,52 & 0,41 & 0,006 \\
\hline Genotipo(G) & 23 & $23,43 \mathrm{~ns}$ & $36,03 \mathrm{~ns}$ & $0,034 \mathrm{~ns}$ & $82,81 \mathrm{~ns}$ & $3,24 * *$ & $1,09 \mathrm{~ns}$ \\
\hline DxG & 23 & $21,09 \mathrm{~ns}$ & $10,68 \mathrm{~ns}$ & $0,016 \mathrm{~ns}$ & $62,73 \mathrm{~ns}$ & 1,49 ns & $0,60 \mathrm{~ns}$ \\
\hline Error b & 92 & 23,15 & 20,47 & 0,020 & 61,41 & 0,41 & 0,75 \\
\hline $\mathrm{CV}$ & & 8,72 & 1,17 & 9,80 & 23,13 & 7,55 & 14,18 \\
\hline F de V & GL & NVP & PVV & $G / V$ & PCGV & REND & \\
\hline Repetición & 2 & 17,42 & 1,79 & 0,002 & 71,45 & & 14896106,5 \\
\hline Densidad(D) & 1 & $584,02 *$ & $1,69 \mathrm{~ns}$ & 0,000 ns & $80,79 \mathrm{~ns}$ & & $3231605,44 *$ \\
\hline Error a & 2 & 25 & 0,62 & 0,003 & 62,08 & & 118157,22 \\
\hline Genotipo(G) & 23 & $34,92 \mathrm{~ns}$ & $5,89 * *$ & $0,010 * *$ & $308,99 * *$ & & $2925747,87 * *$ \\
\hline DxG & 23 & $59,39 * *$ & $1,34 \mathrm{~ns}$ & 0,003 ns & $59,27 \mathrm{~ns}$ & & $2153530,69 *$ \\
\hline Error b & 92 & 29,70 & 1,12 & 0,003 & 67,95 & & 1083644 \\
\hline $\mathrm{CV}$ & & 37,08 & 14,66 & 11,30 & 13,60 & & 37,0067 \\
\hline
\end{tabular}

de bajas a altas densidades de población, se puede observar una leve reducción en los días a floración.

El LV osciló entre 9,28 y 7,50cm (SIXDRC151 y SX3575RC113). El 79\% de los genotipos evaluados mostraron un promedio estadísticamente similar a los testigos San Isidro y Sindamanoy, que alcanzaron 9,07 y $9,0 \mathrm{~cm}$, respectivamente, mientras que $91 \%$ de las líneas igualaron estadísticamente a la variedad Andina $(8,72 \mathrm{~cm})$. Se destaca la línea SIXDRC151, con $9,28 \mathrm{~cm}$, que supera al $47,6 \%$ de las líneas evaluadas (Tabla 3). La similitud observada entre la mayor parte de los genotipos y los testigos Sindamanoy, San Isidro y Andina, se puede explicar, si se tiene en cuenta que las líneas evaluadas se han obtenido por procesos de retrocruzamiento (RC1 y RC2) con dichos testigos y, por tanto, existe una recuperación de las características deseables de estas variedades, en 75 y $87,5 \%$.

Espinosa \& Ligarreto (2005) manifiestan que el largo de vaina está gobernado, en su mayor parte, por efectos aditivos, en consecuencia, son esos efectos los que permiten lograr la mayor similitud de las líneas con los padres recurrentes.

Los resultados, se diferencian del rango obtenido para esta variable, en la evaluación preliminar de las líneas en generación $\mathrm{F} 4$, en donde se observó mayor variabilidad, con promedios entre 7,1 y $9,7 \mathrm{~cm}$ (Tabla 1 ), lo que demuestra que, en la generación F5, hay mayor uniformidad resultante del proceso de selección entre las líneas evaluadas, que se acercan más al parental recurrente en cada cruzamiento, logrando recuperar el largo de vaina deseable, de las variedades mejoradas Andina, San Isidro y Sindamanoy y mayor estabilidad generacional de los materiales, para en esta característica.

En PVV sobresalieron las líneas afila SIXDRC151 y SX3575R$\mathrm{C} 1 \mathrm{X} 1$, con 8,45 y $8,18 \mathrm{~g}$, respectivamente, al igualar a los testigos Sindamanoy, San Isidro y Andina y superar al $33 \%$, de las demás líneas evaluadas. En contraste, las líneas AXDRC24, AXDRC215, SIXDRC154, SIXDRC183, SX3588RC117, SX3568RC128, SX3575RC113 mostraron menores promedios en el peso de las vainas, que sus parentales recurrentes. Es posible, que en la fase anterior (F4), al aplicar el índice de selección, hayan aportado más otras variables diferentes al peso de vainas para la inclusión de estas líneas, en la presente evaluación. Al respecto, Patiño et al. (1997) argumentan que el peso de la vaina depende del tamaño, número, peso y madurez de sus granos; lo anterior, pudo influenciar el comportamiento de las progenies (Tabla 3).

Las líneas SX3575RC112, SX3568RC117, SX3568RC128, SX3575RC113, SX3568RC17, SIXDRC183, SX3568RC11 y SIXDRC166, que corresponden al 38\% de las líneas evaluadas, fueron los de mejor relación $\mathrm{G} / \mathrm{V}$, mostrando valores 
Tabla 3. Comparación de medias de los genotipos de arveja (Pisum sativum L.), para las variables: largo de vaina (LV), peso de vaina verde (PVV), relación grano vaina (G/V) y peso de cien granos verdes (PCGV).

\begin{tabular}{|l|r|r|r|r|}
\hline Genotipo & \multicolumn{1}{|l|}{$\mathbf{L V}(\mathbf{c m})$} & PVV $\mathbf{( g )}$ & PCGV $\mathbf{( g )}$ \\
\hline ANDINA & $8,72 \mathrm{ABCD}$ & $8,11 \mathrm{ABCD}$ & $0,51 \mathrm{BCDEF}$ & $63,08 \mathrm{ABCDEFG}$ \\
\hline AXDRC215 & $8,17 \mathrm{BCDE}$ & $5,36 \mathrm{JJ}$ & $0,52 \mathrm{BCDEEF}$ & $46,04 \mathrm{JI}$ \\
\hline AXDRC24 & $8,20 \mathrm{BCDE}$ & $6,01 \mathrm{GHIJ}$ & $0,50 \mathrm{BCDEF}$ & $45,38 \mathrm{~J}$ \\
\hline SINDAMANOY & $9,07 \mathrm{AB}$ & $8,28 \mathrm{ABC}$ & $0,43 \mathrm{G}$ & $53,19 \mathrm{FGHIJ}$ \\
\hline SAN ISIDRO & $9,00 \mathrm{AB}$ & $8,76 \mathrm{~A}$ & $0,46 \mathrm{FG}$ & $60,95 \mathrm{ABCDEFGH}$ \\
\hline SIXDRC1100 & $8,48 \mathrm{ABCD}$ & $8,07 \mathrm{ABCD}$ & $0,46 \mathrm{EFG}$ & $57,09 \mathrm{DEFGH}$ \\
\hline SIXDRC151 & $9,28 \mathrm{~A}$ & $8,45 \mathrm{AB}$ & $0,52 \mathrm{BCDEF}$ & $66,91 \mathrm{ABCDE}$ \\
\hline SIXDRC154 & $7,88 \mathrm{DE}$ & $6,65 \mathrm{DEFGHI}$ & $0,49 \mathrm{CDEF}$ & $53,02 \mathrm{GHIJ}$ \\
\hline SIXDRC166 & $8,27 \mathrm{BCDE}$ & $7,35 \mathrm{ABCDEFG}$ & $0,55 \mathrm{ABCDE}$ & $66,37 \mathrm{ABCDE}$ \\
\hline SIXDRC183 & $8,35 \mathrm{BCDE}$ & $6,42 \mathrm{EFGHI}$ & $0,56 \mathrm{ABC}$ & $59,22 \mathrm{BCDEFGH}$ \\
\hline SX3568RC11 & $8,02 \mathrm{CDE}$ & $7,48 \mathrm{ABCDEF}$ & $0,56 \mathrm{ABC}$ & $71,21 \mathrm{~A}$ \\
\hline SX3568RC117 & $7,88 \mathrm{DE}$ & $5,91 \mathrm{IHJ}$ & $0,57 \mathrm{AB}$ & $56,2 \mathrm{EFGHI}$ \\
\hline SX3568RC128 & $7,55 \mathrm{E}$ & $6,32 \mathrm{FGHIJ}$ & $0,56 \mathrm{ABC}$ & $62,21 \mathrm{ABCDEFG}$ \\
\hline SX3568RC166 & $8,72 \mathrm{ABCD}$ & $7,88 \mathrm{ABCD}$ & $0,48 \mathrm{CDEFG}$ & $68,14 \mathrm{ABCD}$ \\
\hline SX3568RC17 & $8,45 \mathrm{ABCD}$ & $6,92 \mathrm{CDEFGH}$ & $0,56 \mathrm{ABC}$ & $69,99 \mathrm{AB}$ \\
\hline SX3568RC19 & $8,85 \mathrm{ABC}$ & $7,21 \mathrm{BCDEFGH}$ & $0,53 \mathrm{BCDEF}$ & $59,68 \mathrm{BCDEFGH}$ \\
\hline SX3575RC112 & $8,73 \mathrm{ABCD}$ & $7,47 \mathrm{ABCDEF}$ & $0,60 \mathrm{~A}$ & $64,07 \mathrm{ABCDEFG}$ \\
\hline SX3575RC113 & $7,50 \mathrm{E}$ & $4,99 J$ & $0,56 \mathrm{ABC}$ & $50,68 \mathrm{HIJ}$ \\
\hline SX3575RC120 & $9,00 \mathrm{AB}$ & $7,79 \mathrm{ABCDE}$ & $0,54 \mathrm{BCDEF}$ & $66,70 \mathrm{ABCDE}$ \\
\hline SX3575RC18 & $8,88 \mathrm{ABC}$ & $7,76 \mathrm{ABCDE}$ & $0,52 \mathrm{BCDEF}$ & $64,53 \mathrm{ABCDEF}$ \\
\hline SX3575RC19 & $8,73 \mathrm{ABCD}$ & $7,06 \mathrm{BCDEFGH}$ & $0,47 \mathrm{DEFG}$ & $57,86 \mathrm{CDEFGH}$ \\
\hline SX3575RC1X1 & $8,73 \mathrm{ABCD}$ & $8,18 \mathrm{ABC}$ & $0,48 \mathrm{CDEFG}$ & $63,30 \mathrm{ABCDEFG}$ \\
\hline SXDRC118 & $8,95 \mathrm{AB}$ & $7,85 \mathrm{ABCDE}$ & $0,55 \mathrm{BCDEF}$ & $69,16 \mathrm{ABC}$ \\
\hline SXDRC156 & $8,20 \mathrm{BCDE}$ & $7,21 \mathrm{BCDEFGH}$ & $0,52 \mathrm{BCDEF}$ & $59,31 \mathrm{BCDEFGH}$ \\
\hline
\end{tabular}

mayores a 0,55 y superando significativamente a los testigos Sindamanoy y San Isidro, que no superaron al promedio de 0,46. Por otra parte, se destaca la línea SX3575RC112, con una relación G/V de 0,60 , que fue estadísticamente superior a Andina $(0,51)$ (Tabla 3). Esto manifiesta el potencial de las líneas afila seleccionadas, como producto final para el consumidor y ser consideradas como progenitores, en programas de mejoramiento genético. Con la selección para este carácter, se busca obtener mayor peso de los granos y menor peso de las valvas, que conforman las vainas, aumentando la proporción del efecto vertedero hacia los granos y convirtiéndose en un producto atractivo para el mercado. Una relación G/V alta favorece la industrialización de la arveja. En Chile, Hargous (2001) menciona que el promedio industrial de esta relación, alcanza el valor de 0,7; las líneas afila se acercan a este promedio, importante para industrialización.
Para el PCGV, las líneas SX3568RC11, SX3568RC17, SXDRC118, SX3568RC166, SIXDRC151, con valores entre 71,21 y 66,37g, superaron al testigo Sindamanoy. De estas líneas, tres proceden del parental Sindamanoy y una del mismo parental San Isidro. Lo anterior, puede resultar de la acumulación de genes favorables para este carácter, presentes en los progenitores, permitiendo un efecto genético transgresivo, que condujo a obtener promedios superiores a los parentales. Al respecto, Amin et al. (2010) reportaron que el peso de la semilla en arveja se encuentra entre los caracteres poligénicos de alta heredabilidad y presenta una acción génica de tipo aditivo (Tabla 3).

Por otra parte, los resultados para peso de los granos verdes de las líneas afila difieren con algunos autores, como Mera et al. (1996) y Burstin et al. (2007), quienes argumentan que la disminución en el número de hojas en la planta puede reducir el peso de los granos y otras características del fruto; 
sin embargo, en las líneas afila, evaluadas en la presente investigación, no se afectó el peso de grano, debido, posiblemente, a que se trata de un carácter altamente heredable, controlado por genes independientes al gen af, que se han recuperado del padre recurrente.

Para la interacción DxG, la variable NVP, en la D1, el 28,6\% de las líneas fueron estadísticamente iguales al testigo Andina y el $47,6 \%$, igualaron a San Isidro y Sindamanoy. Las líneas afila SIXDRC1100, SX3568RC19, SX3575RC18, SX3575RC1X1, SX3575RC113 y SX3568RC11 sobresalen por presentar promedios iguales o superiores a 18 vainas por planta (Tabla 4). La línea afila SIXDRC1100, que alcanzó un promedio de 23 vainas por planta, tiene como uno de sus parentales a la variedad San Isidro, resultado que contrasta con lo reportado por Ligarreto \& Ospina (2009), quienes no lograron obtener altos promedios en número de vainas por planta, en generaciones F1 y F2, de cruzamientos simples, realizados con la variedad San Isidro. Esta diferencia en resultados es entendible, si se tiene en cuenta que la línea SIXDRC1100 se obtuvo de un retrocruzamiento con San Isidro, que les permitió recuperar en un $75 \%$ características de esta variedad, entre ellas, su alta expresión en el número de vainas por planta.

Para la densidad D2, no se observaron diferencias significativas entre las líneas y los testigos (Tabla 4). Al respecto, Casanova et al. (2012) determinaron que las densidades mayores a 83.333 y hasta 166.666 plantas ha $^{-1}$ fueron estadísticamente similares para esta variable. El fenómeno anterior, se puede deber a que el aumento en la densidad de población ocasiona mayor competencia entre plantas por luz y temperatura, afectando algunos caracteres, como el número de vainas por planta; esto conlleva a una menor manifestación del potencial genético de algunos materiales y no permite su diferenciación, dentro de la población estudiada. En igual sentido, Azpilicueta et al. (2012) encontraron que, en cultivos a menor densidad, el $80 \%$ de las plantas tuvieron carga en cinco ramas, mientras las de mayor densidad, solo en tres.

En REND, el análisis de varianza mostró significancia para la interacción densidad x genotipo, indicando un comportamiento diferencial de los genotipos, a través de las densidades evaluadas. En D1, se observó que 11 de las 21 líneas afila, con rendimientos entre 4.142 y $2.236 \mathrm{~kg} \cdot \mathrm{ha}^{-1}$, lograron igualar estadísticamente a los testigos Sindamanoy $\left(4.231 \mathrm{~kg} \cdot \mathrm{ha}^{-1}\right)$ y Andina $\left(3.714 \mathrm{~kg} \cdot \mathrm{ha}^{-1}\right)$, entre ellas, sobresalen SIXDRC1100, SX3575RC18, con 4.142 y $4.045 \mathrm{~kg} \cdot \mathrm{ha}^{-1}$, que también fueron estadísticamente similares a la variedad San Isidro $\left(3.694 \mathrm{~kg} \cdot \mathrm{ha}^{-1}\right.$ ) y superaron al $47,62 \%$ de las líneas con gen afila evaluadas (Tabla 4). Lo anterior, es explicable si se tiene en cuenta que las líneas afila evaluadas tienen como uno de los progenitores a las variedades testigo (Andina, San Isidro, Sindamanoy).
La comparación de promedios para D2 muestra el potencial de algunas líneas afila, que superan significativamente en rendimiento a las variedades mejoradas, usadas como testigos. La línea SXDRC118, con 5163kg $\cdot \mathrm{ha}^{-1}$, mostró mayor promedio a los tres testigos y al $66 \%$, de las demás líneas evaluadas, mientras que SX3568RC17, con 4775 $\mathrm{kg} \cdot \mathrm{ha}^{-1}$, logró mejores rendimientos que los testigos Sindamanoy y San Isidro. Por otra parte, las líneas SX3575RC19, SX3575RC1X1 y SX3575RC18, con promedios entre 4775 y $3941 \mathrm{~kg} \cdot \mathrm{ha}^{-1}$, superaron a Sindamanoy. Los resultados indican que, para este tipo de líneas con gen afila, se pueden reducir las distancias de siembra, sin alterar negativamente las condiciones para su crecimiento y desarrollo, permitiendo que las plantas se mantengan erectas, entrelazando sus zarcillos y logrando mejorar su rendimiento en función del mayor número de plantas, por unidad de área.

Según Burstin et al. (2007), el efecto vertedero depende de las condiciones ambientales y en limitaciones de luz, que ocurren con el aumento de la densidad de población, se pueden generar mayores concentraciones de asimilados en otros órganos, diferentes a las hojas. En el mismo sentido, puede ocurrir que, en algunas líneas afila de alto rendimiento, los asimilados que, en plantas convencionales se dirigen hacia las hojas, al ser estas sustituidas por zarcillos, se pueden direccionar parcialmente a otras partes de la planta, como vainas y granos, aportando al rendimiento.

En general, se observó que, mientras en la densidad D1 los testigos Sindamanoy y Andina superaron al $47 \%$ de las líneas afila y San Isidro a dos de las 21 líneas, en la densidad 2, ninguno de los testigos superó en REND a las líneas afila estudiadas, confirmando la mejor respuesta de las líneas afila, al incrementarse la densidad de población. Es posible, que en algunas líneas afila el aumento de la población no produzca una importante reducción en la luz, debido a la transformación de hojas en zarcillos y a la mejor distribución de las demás estructuras de la planta, resultando favorable su respuesta al incremento en la densidad, como se observó en la línea SXDRC118, que pasó de 1767 a $5163 \mathrm{~kg} \cdot \mathrm{ha}^{-1}$, al duplicar su densidad de población. Por el contrario, en las variedades de hojas normales Andina, Sindamanoy y San Isidro, la competencia por luz, pudo incidir en la reducción del rendimiento.

Al respecto, Cousin (1997) afirma que los cambios morfológicos resultantes de reemplazar hojas por zarcillos no están directamente correlacionados con la eficiencia fotosintética de la planta, pues no limita el peso de las semillas ni el rendimiento, además, la disminución de foliolos no es limitante en la asimilación de luz, porque su ausencia permite que el resto de los órganos realice la fotosíntesis, de manera más eficiente. Al reducir el área foliar en un $40 \%$, se presenta mayor asimilación fotosintética de las vainas, logrando mejorar 
Tabla 4. Comparación de medias de los diferentes genotipos de arveja (Pisum sativum L.), para las densidades de población evaluadas para las variables número de vainas por planta (NVP) y rendimiento (REND).

\begin{tabular}{|l|r|r|l|r|r|}
\hline \multicolumn{2}{|c|}{ Número de vainas por planta (NVP) } & \multicolumn{3}{c|}{ Rendimiento REND (kg.ha ${ }^{-1}$} \\
\hline Genotipo & D1 & D2 & Genotipo & D1 & D2 \\
\hline ANDINA & $27 \mathrm{~A}$ & $13 \mathrm{~A}$ & SINDAMANOY & $4231 \mathrm{~A}$ & $1655 \mathrm{E}$ \\
\hline SANISIDRO & $25 \mathrm{AB}$ & $13 \mathrm{~A}$ & SIXDRC1100 & $4142 \mathrm{~A}$ & $2193 \mathrm{CDE}$ \\
\hline SINDAMANOY & $24 \mathrm{AB}$ & $14 \mathrm{~A}$ & SX3575RC18 & $4045 \mathrm{~A}$ & $3941 \mathrm{ABCD}$ \\
\hline SIXDRC1100 & $23 \mathrm{ABC}$ & $19 \mathrm{~A}$ & ANDINA & $3714 \mathrm{~A}$ & $2936 \mathrm{BCDE}$ \\
\hline SX3568RC19 & $21 \mathrm{ABCD}$ & $14 \mathrm{~A}$ & SANISIDRO & $3694 \mathrm{AB}$ & $2559 \mathrm{CDE}$ \\
\hline SX3575RC18 & $20 \mathrm{ABCD}$ & $10 \mathrm{~A}$ & SX3575RC1X1 & $3524 \mathrm{AB}$ & $4091 \mathrm{ABCD}$ \\
\hline SX3575RC1X1 & $21 \mathrm{ABCDE}$ & $13 \mathrm{~A}$ & SX3575RC19 & $3341 \mathrm{ABC}$ & $4258 \mathrm{ABC}$ \\
\hline SX3575RC113 & $18 \mathrm{ABCDE}$ & $18 \mathrm{~A}$ & SX3568RC17 & $3268 \mathrm{ABCD}$ & $4775 \mathrm{AB}$ \\
\hline SX3568RC11 & $18 \mathrm{ABCDE}$ & $9 \mathrm{~A}$ & SX3568RC19 & $3257 \mathrm{ABCD}$ & $2852 \mathrm{CDE}$ \\
\hline SX3568RC166 & $16 \mathrm{BCDE}$ & $13 \mathrm{~A}$ & SX3568RC11 & $3176 \mathrm{ABCD}$ & $3512 \mathrm{ABCDE}$ \\
\hline SX3568RC17 & $16 \mathrm{BCDE}$ & $17 \mathrm{~A}$ & SIXDRC166 & $2590 \mathrm{ABCD}$ & $2461 \mathrm{CDE}$ \\
\hline AXDRC24 & $16 \mathrm{BCDE}$ & $19 \mathrm{~A}$ & AXDRC24 & $2511 \mathrm{ABCD}$ & $2272 \mathrm{CDE}$ \\
\hline SIXDRC183 & $16 \mathrm{BCDE}$ & $10 \mathrm{~A}$ & SIXDRC151 & $2505 \mathrm{ABCD}$ & $2764 \mathrm{CDE}$ \\
\hline SIXDRC154 & $15 \mathrm{CDE}$ & $13 \mathrm{~A}$ & SX3568RC117 & $2236 \mathrm{ABCD}$ & $3522 \mathrm{ABCDE}$ \\
\hline SX3575RC19 & $15 \mathrm{CDE}$ & $10 \mathrm{~A}$ & SIXDRC183 & $1891 \mathrm{BCD}$ & $2514 \mathrm{CDE}$ \\
\hline SIXDRC151 & $14 \mathrm{CDE}$ & $19 \mathrm{~A}$ & SX3575RC120 & $1889 \mathrm{BCD}$ & $3134 \mathrm{BCDE}$ \\
\hline AXDRC215 & $13 \mathrm{CDE}$ & $12 \mathrm{~A}$ & SX3568RC128 & $1870 \mathrm{BCD}$ & $2283 \mathrm{CDE}$ \\
\hline SX3568RC128 & $13 \mathrm{CDE}$ & $12 \mathrm{~A}$ & SX3575RC112 & $1861 \mathrm{BCD}$ & $2342 \mathrm{CDE}$ \\
\hline SIXDRC166 & $13 \mathrm{DE}$ & $15 \mathrm{~A}$ & SX3568RC166 & $1860 \mathrm{BCD}$ & $1936 \mathrm{E}$ \\
\hline SX3575RC112 & $13 \mathrm{DE}$ & $8 \mathrm{~A}$ & SX3575RC113 & $1826 \mathrm{BCD}$ & $2159 \mathrm{DE}$ \\
\hline SX3575RC120 & $13 \mathrm{DE}$ & $10 \mathrm{~A}$ & SXDRC118 & $1767 \mathrm{BCD}$ & $5163 \mathrm{~A}$ \\
\hline SXDRC156 & $13 \mathrm{DE}$ & $10 \mathrm{~A}$ & AXDRC215 & $1766 \mathrm{BCD}$ & $3141 \mathrm{BCDE}$ \\
\hline SX3568RC117 & $12 \mathrm{DE}$ & $8 \mathrm{~A}$ & SIXDRC154 & $1514 \mathrm{CD}$ & $2248 \mathrm{CDE}$ \\
\hline SXDRC118 & $10 \mathrm{E}$ & $10 \mathrm{~A}$ & SXDRC156 & $1433 \mathrm{D}$ & $2389 \mathrm{CDE}$ \\
\hline
\end{tabular}

+ Promedios con la misma letra no presentan diferencias significativas $(\alpha=0,05)$.

D 1 = Corresponde a la densidad de 83.333 plantas ha $^{-1}$

D 2 = Corresponde a la densidad de 166.666 plantas $\cdot \mathrm{ha}^{-1}$

la penetración de luz, a través del dosel y disminuyendo la competencia entre plantas, que ocurre cuando hay demasiada producción de biomasa.

En conclusión, la presente investigación permitió identificar líneas afila que igualan o superan a las variedades de hoja convencional Andina, San Isidro y Sindamanoy, en las variables LV, PVV, PCGV, NVP y REND. De igual manera, en la ma- yor densidad D2 (166666plantas·ha ${ }^{-1}$ ), las líneas de arveja, con gen afila, constituyeron una buena opción productiva para rendimiento en vaina verde, destacándose SXDRC118, que superó a los tres testigos, en 75 a 211\%; SX3568RC17, que mostró mayor promedio sobre San Isidro y Sindamanoy, en 86 y $188 \%$ y las líneas SX3575RC18, SX3575RC1X1 y SX3575RC19, sobre Sindamanoy, en 138 a $157 \%$. 
Agradecimientos: Los autores agradecen a la Universidad de Nariño, en especial a los integrantes del grupo de cultivos andinos (Gricand), al programa de jóvenes investigadores de Colciencias y al centro internacional de agricultura limpia-Lope del Sena. Conflicto de intereses: El manuscrito fue preparado y revisado con la participación de todos los autores, quienes declaramos que no existe conflicto de intereses que ponga en riesgo la validez de los resultados presentados. Financiación: Este estudio fue financiado por Colciencias y la Universidad de Nariño.

\section{REFERENCIAS}

1. AMIN, A.; MUSHTAQ, F.; SINGH, P.; WANI, P.; SPALDON, S; NAZIR, N. 2010. Genetics and breeding of pea. International J. Current Research (IJCR). 10:28-34.

2. AZPILICUETA, M.; IRIGOYEN, I.; LASA, B.; MURO, J.; APARICIO, T. 2012. Yield and quality of sugar snap pea in the Ebro Valley: Sowing date and seed density. Sci. Agric. (Brazil). 69(5):320-326. http://dx.doi. org/10.1590/S0103-90162012000500006

3. BURSTIN, J.; MARGET, P.; HUART, M.; MOESSNER, A.; MANGIN, B.; DUCHENE, C.; DESPREZ, B.; MUNIER, N.; DUC, G. 2007. Developmental Genes Have Pleiotropic Effects on Plant Morphology and Source Capacity, Eventually Impacting on Seed Protein Content and Productivity in Pea. American Society of Plant Biologists (ASPB). (Francie).144:768-781. https://doi.org/10.1104/pp.107.096966

4. CASANOVA, L.; SOLARTE, J.; CHECA, O. 2012. Evaluación de cuatro densidades de siembra en siete líneas promisorias de arveja arbustiva (Pisum sativum L.). Rev. Ciencias Agrícolas. (Colombia). 29(2):129-140.

5. CHECA, O.; BASTIDAS, J.; NARVÁEZ, O. 2017. Evaluación agronómica y económica de arvejas arbustiva (Pisum sativum L.) en diferentes épocas de siembra y sistema de tutorado. Rev. U.D.C.A Act. \& Div. Cient. (Colombia). 20(2):279-288.

6. CHECA, O.; RODRÍGUEZ, M. 2015. Resistencia a oídio Erysiphe polygoni y rendimiento en arveja afila Pisum sativum L. Temas Agrarios. (Colombia). 20(2):58-71. https://doi.org/10.21897/rta.v20i2.759

7. CHECA, O. 2014. Investigación para el mejoramiento de la tecnología de producción de arveja (Pisum sativum L.) en el departamento de Nariño. Disponible desde Internet en: http://www.ucc.edu.co/pasto/prensa/2014/Documents/1Resumen_Proyecto_L\%ADneas\%20Arveja.pdf (con acceso $\overline{15} / 10 / 2014$ ).
8. CHECA, O. 1994. La arveja y sus sistemas de cultivo. Corpoica. Boletín divulgativo No. 104. San Juan de Pasto. 11p.

9. COUSIN, R. 1997. Pea (Pisum sativum L.). INRA, Station de Gentique et d' Amélioration des Plants, Versailles, France. Field Crops Res. (Francia). 53:111-130 p.

10. COTE, R.; GERRATH, J.; PETERSON, C.; GRODZINSKI, G. 1992. Sink to source transition in tendrils of a semileafless utant, Pisum sativum cv Curly. Plant Physiol. (Canadá). 100:1640-1648. https://doi. org/10.21897/rta.v20i2.759

11. DORDEVIĆ, R.; ZDRAVKOVIĆ, J.; ZEČEVIĆ, B.; CVIKIĆ, D.; IVANOVIĆ, M. 2004. Correlation of yield and yield components for afila and normal leave pea (Pisum sativum L.). Genetika. 36(1):39-45.

12. ESPINOSA, N.; LIGARRETO, G. 2005. Evaluación de la habilidad combinatoria y heterosis de siete progenitores de arveja Pisum sativum L. Agron. Colomb. (Colombia). 23(2):197-206.

13. FALLOON, R.; MCERLICH, A.; SCOTT, R. 1989. Powdery mildew of peas; possible causes of recent epidemics and prospects for control. Proceedings of the 42, New Zealand Weed, and Pest Control Conference. p.247-250.

14. FEDERACIÓN NACIONAL DE CULTIVADORES DE CEREALES Y LEGUMINOSAS-FENALCE. 2010. El cultivo de arveja historia e importancia. Importancia de los cultivos representados por Fenalce. El Cerealista. p.32-34.

15. GIACONI, V.; ESCAFE, M. 1988. Cultivo de hortalizas. Editorial Universitaria. Décimo quinta versión. 45p.

16. GONZÁLEZ, F.; LIGARRETO, G. 2006. Rendimiento de ocho genotipos promisorios de arveja arbustiva (Pisum sativum L.) bajo sistema de agricultura protegida. Fitotecnia Colombiana. (Colombia). 6(2):52-61.

17. HARGOUS, F. 2001. Sensorial and agronomical evaluation of seven pea's cultivars (Pisum sativum L.) for IQF freezing. FAO. Agris. 92p.

18. HOFER, J.; TURNER, L.; HELLENS, R.; AMBROSE, M.; MATTHEWS, P.; MICHAEL, A.; ELLIS, N. 2009. Tendril-less Regulates Tendril Formation in Pea Leaves. The plant cell. (Estados Unidos). 21:420-428. https://doi.org/10.1105/tpc.108.064071 
19. HOFER, J.; TURNER, L.; HELLENS, R.; AMBROSE, M.; MATTHEWS, P.; MICHAEL, A.; ELLIS, N. 1997. Unifoliate regulates the morphogenesis of the leaf and flower in the pea. Current biology. (Inglaterra). 7(8):581-587.

20. KHVOSTOVA, V. 1983. Genetics and breeding of peas. USDA, Springfield, IL. p.12-96.

21. LIGARRETO, G.; OSPINA, A. 2009. Análisis de parámetros heredables asociados al rendimiento y precocidad en arveja voluble (Pisum sativum L.) tipo Santa Isabel. Agron. Colom. (Colombia). 27(3):333-339.

22. MERA, M.; LEVIO, J.; ALCALDE, J.; MORALES, M.; GALDAMES, G. 1996. Brisca INIA, primera variedad de arveja afila obtenida en Chile. Agricultura técnica. (Chile). 56(4):282-286.

23. ORBES, A.; BECERRA, J. 1982. Control químico de enfermedades foliares de arveja (Pisum sativum L.) en el altiplano de Pasto, departamento de Nariño. Universidad de Nariño. Facultad de Ciencias Agrícolas. (Colombia). 37p.
24. PESIC, V.; DJORDJEVIC, R.; KADHUM, E.; JANKOVIC, P.; MISIC, D. 2013. Influence of the afila gene on grain yield in pea (Pisum sativum L.). Bulgarian $\mathrm{J}$. Agr. Science (BJAS). (Inglaterra). 19(2):186-193.

25. PACHECO, C.; VERGARA, H.; LIGARRETO, G. 2011. Clasificación de 42 Líneas Mejoradas de Arveja (Pisum sativum L.) por Caracteres Morfológicos y Comportamiento Agronómico. Rev. Fac. Nac. Agron. Medellín. (Colombia). 63(2):5543-5553.

26. PATIÑO, W.; VALDERRAMA, J.; ÑUSTEZ, C. 1997. Evaluación de nueve variedades de arveja (Pisum sativum L.) para uso industrial, en la región de Suba. Agron. Colom. (Colombia). 19(2):108-118.

Recibido: Febrero 22 de 2018

Aceptado: Octubre 6 de 2018

\section{Cómo citar:}

Riascos Delgado, M.E.; Checa Coral, O.E. 2018. Evaluación y selección de líneas de arveja con gen afila bajo dos densidades de población. Rev. U.D.C.A Act. \& Div. Cient. 21(2):367-376. https://doi.org/10.31910/rudca.v21.n2.2018.984 